\title{
Armement des hommes de couleur et liberté aux Antilles : le cas de la Guadeloupe pendant l'Ancien régime et la Révolution
}

Frédéric Régent

\section{(2) OpenEdition}

Journals

Édition électronique

URL : https://journals.openedition.org/ahrf/9023

DOI : 10.4000/ahrf.9023

ISSN : 1952-403X

Éditeur :

Armand Colin, Société des études robespierristes

Édition imprimée

Date de publication : 1 juin 2007

Pagination : 41-56

ISSN : 0003-4436

\section{Référence électronique}

Frédéric Régent, «Armement des hommes de couleur et liberté aux Antilles : le cas de la Guadeloupe pendant l'Ancien régime et la Révolution », Annales historiques de la Révolution française [En ligne], 348 | Avril-Juin 2007, mis en ligne le 01 juin 2010, consulté le 28 avril 2022. URL : http:// journals.openedition.org/ahrf/9023; DOI : https://doi.org/10.4000/ahrf.9023

Ce document a été généré automatiquement le 28 avril 2022.

Tous droits réservés 


\title{
Armement des hommes de couleur ${ }^{1}$ et liberté aux Antilles : le cas de la Guadeloupe pendant l'Ancien régime et la Révolution
}

\author{
Frédéric Régent
}

Défendre la colonie pour obtenir individuellement la libertéUn usage d'Ancien Régime

1 Le système esclavagiste face à certaines nécessités s'assouplit relativement vite. Pour des nécessités de politique extérieure et de défense de l'île, le recours à des libres de couleur ou esclaves armés est préconisé. La protection de la Guadeloupe est assurée par des troupes réglées venues de France ${ }^{3}$, mais aussi des compagnies de milice composées de la population libre masculine de l'île. Dans la compagnie du quartier de Ferri à Deshaies, des esclaves noirs ont été recrutés pour compléter les effectifs car la région est très peu peuplée, selon le témoignage de Labat lors de sa visite en Guadeloupe en 16964. En 1703, les esclaves participent à la défense de la colonie contre les Anglais. Au départ, ils sont réquisitionnés pour effectuer des travaux de terrassement et de charroi pour les fortifications. Mais, en 1759, la colonie a utilisé pour lutter contre l'attaque britannique des esclaves armés. Pendant la guerre de Sept Ans, le mulâtre Louison fut capitaine d'une des trois compagnies d'esclaves créées en 1759. Sa bravoure et son dynamisme lui valurent d'être affranchi par son maître le gouverneur Nadau du Treil ${ }^{5}$. Dans l'article 11 de la capitulation française de la Guadeloupe signée le 2 mars 1759, il est stipulé que les esclaves ayant porté des armes conserveront leur liberté. L'idée qu'un esclave ayant porté les armes devienne libre s'est peu à peu imposée ${ }^{6}$. En 1763, la milice est supprimée ${ }^{7}$. Mais elle est rétablie par une ordonnance royale le 1er septembre 1768, avec un nouveau règlement. Il prévoit la division de la colonie en quartiers. Chaque quartier est commandé par un commandant de quartier qui dirige l'ensemble des compagnies. Chacune d'elle est formée d'un capitaine, d'un lieutenant, d'un sous-lieutenant, de deux sergents, de quatre caporaux, de quarante-six fusiliers et d'un tambour nègre ou mulâtre aux frais du capitaine. Les compagnies blanches sont composées de tous les hommes de 15 à 55 ans et celles de couleur de tous les libres ou 
affranchis de 15 à 60 ans. Les deux classes juridiques servent dans des compagnies distinctes. Cependant, tous les officiers sont blancs. Les compagnies de couleur ont le même armement et sont soumises à la même discipline et aux mêmes revues que les compagnies des blancs. Leurs bas officiers sont choisis parmi les gens de couleur par les capitaines. Le rôle des compagnies de couleur est la chasse des nègres marrons, des déserteurs et la police des quartiers ${ }^{8}$. Des états fournis par l'administration coloniale au ministre de la Marine et des Colonies fournissent des renseignements sur le nombre de libres de couleur incorporés dans les compagnies de milice.

Effectifs des milices entre 1768 et $1788^{9}$

\begin{tabular}{|l||l|l|l|l|l|l|}
\hline Quartier de milice & \multicolumn{2}{|l|}{1768} & \multicolumn{2}{l|}{1778} & \multicolumn{2}{l|}{1788} \\
\hline & Gens de couleur & Total & Gens de couleur & Total & Gens de couleur & Total \\
\hline \hline Ensemble & 536 & 2885 & 778 & 3398 & 1119 & 4442 \\
\hline
\end{tabular}

2 La proportion de libres de couleur dans la milice passe de $19 \%$ en 1768 à $25 \%$ en 1788 . Nous observons un doublement en 20 ans du nombre des libres de couleur dans la milice. Cette forte augmentation du nombre de libres de couleur n'est pas seulement imputable à une croissance démographique de cette catégorie de population. L'étude des actes d'affranchissement permet de comprendre cette progression. Les maîtres peuvent autoriser certains de leurs esclaves en état de porter les armes à servir dans la milice pendant une certaine période, au terme de laquelle ils obtiennent l'affranchissement de droit sans avoir à payer aucune taxe ou la capitation annuelle. Les aspects financiers de cette pratique sont attrayants pour la colonie, les maîtres et les esclaves. Ces derniers contribuent à l'intérêt de la première en la défendant et les seconds peuvent accorder la liberté sans aucun frais. L'essentiel de l'augmentation des miliciens de couleur provient d'esclaves affranchis par le service militaire.

3 L'entrée dans la milice est donc un moyen d'obtenir la liberté. Comme dans la Rome antique, le service des armes est un moyen de sortir de l'esclavage. Pour y parvenir, il faut tout d'abord une permission écrite du maître passée devant notaire et l'accord du commandant du district des gens de couleur. L'entrée dans la milice donne la liberté de fait. Après un temps de service ramené à 8 ans en 1792 et l'autorisation des administrateurs, le milicien obtient son acte d'affranchissement. Ainsi, Célestin reçoit le consentement de son maître pour qu'il «se fasse incorporer dans la compagnie des gens de couleur libres de la paroisse de la Basse-Terre, pour y faire son service, comme les affranchis de cette île le font, sous le bon plaisir de Monseigneur le général et de $\mathrm{M}$. Charles Chabert Delisle, commandant de milice; afin que par la suitte, le dit Célestin par son zèle et son exactitude à son service, puisse parvenir à mériter son affranchissement de la bienfaisance du gouvernement ». Charles Chabert Delisle donne son accord à l'entrée de Célestin dans sa compagnie et signe l'acte ${ }^{10}$. L'enrôlé devient alors libre de fait. Il doit par conséquent être inscrit sur le dénombrement de son ancien propriétaire.

4 Le délai entre l'entrée dans le district des gens de couleur et celui de l'obtention de l'affranchissement est long. Ainsi, le maître du mulâtre François déclare que celui-ci 
sert dans la milice depuis plus de seize ans et qu'il a mérité depuis longtemps un titre légal de liberté1 ${ }^{11}$ À la fin de ce temps, les miliciens reçoivent un certificat attestant de leur service. L'obtention de la liberté par la voie de l'enrôlement dans la milice est faite pour ceux qui ne veulent ou ne peuvent pas payer les frais d'affranchissement. C'est surtout à partir des années 1780 que nous trouvons dans les actes notariés les mentions d'esclaves que leur maître autorise à servir dans la compagnie des gens de couleur libres. Nous observons donc là une procédure et un mode de recrutement tout à fait spéciaux, pendant une douzaine d'années, à la fin de l'Ancien Régime. De son plein gré et sans compensation financière, autre que l'exemption du paiement de la capitation, le maître autorise l'esclave à s'engager dans la milice. Pareilles conditions expliquent que la procédure ait été utilisée par des maitres aux moyens financiers modestes : femme, propriétaire de couleur, religieux agissant en leur nom personnel, mais désireux d'affranchir leur esclave. Ils font, en effet, l'économie de la taxe à verser à la caisse des Libertés. Toutefois, des liens de service et d'obligation noués avec l'esclave et dont la minute ne livre rien, ont pu par avance compenser le rachat et amplement justifier, du point de vue du maitre, l'autorisation à rejoindre la milice ${ }^{12}$.

5 Lors de la déclaration de guerre à l'Angleterre, en 1778, le gouvernement sollicite la participation accrue des hommes de couleur à la défense militaire. Un corps de "volontaires libres de la Guadeloupe » qui doit permettre de tenir bon en cas de siège, en attendant des renforts d'Europe est créé le 28 août 1782. 527 hommes de couleur considérés comme des troupes réglées vont servir dans ce corps. Il est toujours commandé par des blancs. Entre 1782 et 1785, cette nouvelle force représente en Guadeloupe un effectif moyen de 300 hommes sous les armes ${ }^{13}$. Les hommes de couleur ne sont plus considérés comme des auxiliaires ou une force de police, mais comme une véritable unité militaire de combat. Un colon écrit que ce corps des Volontaires libres de la Guadeloupe, formé de l'élite des gens de couleur, manœuvre aussi bien que les troupes réglées ${ }^{14}$. Certains membres de ce corps vont jouer un rôle important dans la Révolution.

6 La création de ce "bataillon des Volontaires libres" entraîne une diminution des effectifs d'hommes de couleur servant dans la milice dans son organisation traditionnelle. Au moment de la guerre d'indépendance américaine et dans les années qui suivent, les deux tiers des hommes de couleur servent dans le bataillon des volontaires libres. Un minimum de 240 hommes doit servir de force de police alors que le reste est enrôlé dans le bataillon. Celui-ci est dissous peu après la signature de la paix. La colonie retrouve une organisation de sa milice comparable à celle de 1768 .

7 En 1788, 1225 hommes de couleur de 15 à 60 ans appartiennent aux compagnies de milice. Un quart de la milice est formée par des gens de couleur mais, dans certaines paroisses, le tiers est dépassé. Nous avons peu d'indications sur la durée du service dans la milice au cours d'une année. Nous savons cependant, que les hommes doivent fournir leur équipement. De ce fait, chaque homme de couleur libre est armé et dispose d'un fusil. D'ailleurs, les contrats de mariage prévoient en préciput la conservation des armes pour les époux lors du décès de leur femme. Les hommes de couleur apprécient particulièrement le fait d'être armés comme le précise Moreau de Saint-Méry.

«Les gens de couleur libres sont aussi assujettis à être armés comme les blancs; mais indépendamment d'une espèce d'amour qu'ils mettent à avoir de bonnes armes ils y sont interessés par le besoin qu'ils en ont dans les chasses de nègres marons auxquelles on les employe $»^{15}$. 

de couleur ont un rôle paradoxal car elles ont à la fois une fonction de maintien du système esclavagiste et d'affranchissement des esclaves bénéficiant des faveurs de leur maître. Entre 1768 et 1785 , le souci d'améliorer la défense de l'île engage les administrateurs à encourager l'affranchissement à l'issue de plusieurs années de service dans les milices des esclaves exerçant un métier ${ }^{16}$. Le contexte de la rivalité franco-anglaise à la fin du XVIII siècle favorise les émancipations individuelles. John Garrigus est le premier à avoir attiré l'attention sur l'évolution profonde et rapide de la caste des gens de couleur qui a causé la déstabilisation de la société esclavagiste de Saint-Domingue après 1760 . Cet historien a en particulier décrit les responsabilités croissantes confiées aux milices de couleur durant la guerre d'Indépendance ${ }^{17}$. L'ensemble de ce processus crée une culture de l'arme et donne naissance à une mentalité militaire dans la population de couleur libre et dans une moindre mesure servile, bien avant la Révolution.

L'armement massif des esclaves en question au début de la Révolution

Le 26 janvier 1790, sur la proposition de Coquille Dugommier, la milice nationale remplace les anciennes milices ${ }^{18}$. Son organisation est fixée par le règlement du 14 février, les gens de couleur sont toujours établis dans des unités distinctes des blancs ${ }^{19}$. Le 2 décembre 1790, une garde municipale est établie dans chaque paroisse. Cette garde, organisée en brigades, est composée de blancs et de gens de couleur libres, avec au moins un blanc pour trois hommes de couleur. Ces derniers, âgés de 15 à 50 ans, doivent, à tour de rôle, une année de service à la garde municipale. Ceux dont le métier rend la chose trop gênante peuvent payer un remplaçant ${ }^{20}$. Les gardes municipales ainsi formées sont prévues pour être composées d'une majorité d'hommes de couleur et avoir une fonction de simple police. À la fin de 1791, dans la plupart des paroisses, les libres de couleur sont écartés de la garde nationale et ne servent que dans les gardes municipales $^{21}$. À la fin de septembre 1791, les libres de couleur signent une pétition pour demander leur admission dans la garde nationale ${ }^{22}$. Ils se rendent compte que leur accès à cette dernière peut être un pas décisif pour leur entrée dans la citoyenneté.

En septembre 1792, la Guadeloupe est entrée dans la contre-révolution. Elle a besoin de troupes pour repousser les gardes nationales patriotes expédiées de France. Certains capitaines de milices contre-révolutionnaires décident de faire entrer dans leur compagnie un certain nombre d'esclaves, mais l'assemblée coloniale royaliste interdit ce type d'enrôlement le 27 novembre $1792^{23}$. Cependant, elle décide, le 30 novembre 1792, que «tout homme de couleur qui prouvera légalement avoir fait un service militaire pendant huit ans, est déclaré, dès à présent, avoir acquis la liberté ${ }^{24}$. Un certain nombre de libres de fait deviennent affranchis de droit. Elle complète cette mesure le 1er décembre 1792 en créant un corps de volontaires sous la dénomination de bataillon de volontaires libres de la Guadeloupe. Ce bataillon est formé d'hommes libres de couleur. Ceux servant dans les milices pour parvenir à la liberté qui n'ont pas encore accompli les huit ans de service sont tenus de s'enrôler dans ce bataillon afin de compléter le temps qui leur manque. Il leur est fourni un uniforme et une solde de 5 sous par jour pour les fusiliers, 10 pour les caporaux et 15 pour les sergents. Ce bataillon est "à la disposition de $\mathrm{M}$. le gouverneur et à la solde de la colonie $»^{25}$. Le gouverneur d'Arrot crée un corps de volontaires similaire à celui formé pendant la guerre d'Indépendance américaine. Grâce à cette création, il espère rassembler en une unité de combat des compagnies dispersées dans toute la colonie. Il rassemble ses 
troupes, le 28 décembre 1792, au camp de Saint-Jean à Petit-Bourg. Deux jours plus tard, la petite armée royaliste panique à la vue des soldats républicains et se disperse sans combattre ${ }^{26}$. L'idée d'armer les esclaves commence à germer dans certains esprits, mais les royalistes par crainte d'effets négatifs ont refusé d'envisager leur recrutement de manière générale. Ils ont accepté les enrôlements au cas par cas de "bons sujets » certainement déjà destinés à l'affranchissement.

11 La question de l'organisation de l'armée et de l'armement de la population de couleur se pose à nouveau, lorsque la nouvelle de la déclaration de guerre entre la France et le Royaume-Uni le 1er février 1793 est connue en Guadeloupe le 18 mars. À cette époque, le nouveau gouverneur Collot affirme ne disposer que de 144 hommes du régiment de la Guadeloupe et 27 du bataillon du Forez ${ }^{27}$. Cette force armée qui s'élevait à 1200 hommes en 1789 a fortement diminué à cause des décès, des désertions, des proscriptions frappant les soldats patriotes, puis de l'émigration des militaires royalistes. L'état de la garde nationale ne semble être guère meilleur. Le 26 mars 1793, Collot se rend compte, lors de la revue de la garde nationale de Gosier, qu'il y a beaucoup de citoyens de couleur, mais que plusieurs d'entre eux sont sans arme faute de moyens ${ }^{28}$. Cependant, pour la première fois, ces derniers servent dans les mêmes compagnies que les citoyens blancs. Cet amalgame met fin à la ségrégation qui existait dans les anciennes milices. Les hommes de couleur sont minoritaires dans la garde nationale de Petit-Canal. Ils sont 43 sur 177 , ce qui fait une proportion de $24 \%{ }^{29}$. Par rapport aux chiffres de 1788 (16\%, 33 sur 201) nous observons une progression de la part des citoyens de couleur qui peut s'expliquer par les autorisations données par les patriotes à certains de leurs esclaves pour servir dans la garde nationale et obtenir la liberté, mais aussi par la diminution du nombre de blancs, liée à l'émigration des royalistes.

Le 10 avril 1793, le gouverneur propose pour défendre la colonie d'organiser quatre bataillons soldés ${ }^{30}$. Collot préconise un large emploi des citoyens de couleur dans la force armée. Mais la commission n'accepte la création que d'un seul bataillon. Celui-ci se forme à partir de mai 1793 d'après les premières nominations d'officiers effectuées ce mois-là. Le mulâtre tailleur d'habit François Mondésir Gripon est le premier homme de couleur à devenir officier de la garde nationale en Guadeloupe. Son frère Louison l'est également. La plupart des citoyens de couleur nommés officiers sont des libres de couleur qui se sont signalés dans la période précédente par leur dynamisme économique et leur sociabilité au sein de leur caste en étant parrain d'enfant ou témoin lors des mariages ou des inhumations.

Le recrutement des volontaires de la garde nationale s'effectue parmi les blancs, les nouveaux citoyens, mais aussi parmi les esclaves. Le citoyen Jean Louis Taillandier de Vieux-Habitants, voulant récompenser le mulâtre Charles, âgé de 23 ans, et « désirant donner un témoignage de son patriotisme et procurer à la République un sujet qui puisse lui être utile dans ce moment de crise ", l'autorise à s'engager dans la garde nationale ${ }^{31}$. C'est la continuation du procédé d'affranchissement par la milice observé sous l'Ancien Régime. Cependant, l'esclave incorporé ne devient pas tout de suite citoyen.

«Considérant que les gens de couleur qui doivent servir la patrie pendant un espace de tems qui a été réduit à huit années, jouissent provisoirement des droits civils, mais n'ont point encore acquis les droits politiques appartenans à la liberté pour l'obtention de laquelle ils font un service qui est le plus beau titre pour y parvenir $[. ..] \|^{32}$. 
Cette décision entraîne la création d'une nouvelle classe d'hommes qui ne sont plus esclaves, car ils ont des droits civils, mais qui ne sont pas encore citoyens car ne bénéficiant pas de droits civiques, lesquels sont accordés à tout esclave ayant servi huit ans dans la milice ou la garde nationale, le 18 mai $179^{33} 3$. Les recrutements d'esclaves restent très limités et ne concernent que des individus bénéficiant déjà d'une forte autonomie d'action dans le cadre de la liberté de fait. Une utilisation importante des esclaves est cependant préconisée pour les travaux de réparation et d'entretien des fortifications et des batteries de la colonie. Le 5 avril 1793, Collot demande 300 esclaves pour cette tâche ${ }^{34}$. Une autre disposition va plus loin dans l'usage militaire de la population servile. Cet arrêté permet la mise à disposition pour la défense de la colonie de 50 esclaves dans chacune des 31 paroisses, soit un total de 1550. Ils ne sont pas armés, mais ils sont destinés à suivre de près les opérations militaires ${ }^{35}$. Le 1er août 1793 , les troupes réglées de ce qui reste du régiment de Guadeloupe sont amalgamées à la garde nationale et forment deux compagnies indemnisées ${ }^{36}$. En janvier 1794, face à la menace anglaise, Collot décide de former un second bataillon de gardes nationales indemnisées. Il s'agit de recruter 500 esclaves à raison d'un esclave mâle adulte sur 10 ou 15 selon le type d'habitation ${ }^{37}$. Si les soldats sont esclaves, les officiers sont tous citoyens, dont beaucoup de couleur. Cependant, la mesure a de nombreux détracteurs. Des 500 esclaves prévus, seulement 200 sont levés. Parmi ces derniers, toujours selon Collot, la plupart sont infirmes ou ont reçu l'ordre de leur maitre de ne pas combattre ${ }^{38}$. L'exemple de la révolte de Saint-Domingue est mis en exergue par les sans-culottes pour dénoncer le danger d'une telle mesure. Pourtant celle-ci a ses chauds partisans notamment au sein de la société populaire de Basse-Terre, plus modérée. Le 5 février 1794, le citoyen Lacharière-Larery fait valoir à la tribune de la Société que le seul moyen de sauver la République est d'armer les esclaves: "Créons des nouveaux défenseurs à la liberté » déclare-t-il. Il propose que le service armé transforme les esclaves en citoyens.

«On ne peut donner la liberté politique, dira-t-on, à des gens qui n'ont rien fait pour l'obtenir ; non sans doute, mais on peut leur en montrer l'expectative et leur faciliter les moyens d'y arriver. Que celui qui pourra montrer trois blessures soit libre à l'instant, que celui qui aura fait tomber trois ennemis soit libre, que celui qui aura sauvé la vie d'un citoyen soit réputé sur le champ citoyen lui-même ; enfin, la récompense de telles actions et des vertus en tout genre, vous offrira les moyens d'éveiller l'honneur dans l'âme de ces hommes nouveaux, et de les préparer à être admis dans la grande classe des hommes libres [...] Pour faire utilement la guerre, il faut deux choses, des hommes et de l'argent, des hommes nous pouvons en faire $»^{39}$.

Lors de l'attaque anglaise qui survient le 11 avril 1794, la colonie de Guadeloupe ne dispose pas d'une armée capable de résister. Le 20 avril 1794, la capitulation est signée entre Collot et le général Grey. Elle stipule que les troupes françaises se rendront avec les honneurs de la guerre et pourront se rendre en France à condition de ne plus combattre le Royaume-Uni pendant la durée de la présente guerre. En outre, la liberté des esclaves engagés dans la force armée n'est pas ratifiée et ils sont rendus à leurs maîtres ${ }^{40}$.

Au-delà des divisions entre factions politiques rivales, la perte de la Guadeloupe s'explique par la peur d'armer massivement les esclaves. En prenant une demi-mesure, Collot s'est aliéné les plus fervents partisans du maintien de l'esclavage, sans pour autant mobiliser les masses serviles en faveur de la République. Pour les mêmes raisons, la Martinique et une partie de Saint-Domingue sont occupées par les 
Britanniques. En mai 1793, les Anglais ont été repoussés de la Martinique par des citoyens de couleur et des esclaves armés et affranchis aussitôt grâce à Joseph Leborgne $^{41}$ qui seconde Rochambeau. L'armement d'esclaves aurait-il permis de préserver les colonies des Iles du Vent? Leborgne répond affirmativement. En octobre 1794, il publie une brochure, Enfin la vérité sur les colonies, dans laquelle il explique que si les Anglais ont triomphé, c'est que la seconde fois, on n'a pas voulu recourir aux Noirs, que l'on a fait courir le bruit que la Convention se prononçait sur le maintien de l'esclavage. Il ajoute: "Je déclare que si je m'y étais trouvé en 1794, les Anglais n'y auraient trouvé que des cendres et des hommes libres $»^{42}$. En Guadeloupe, l'échec de l'armée de couleur se traduit par le fait qu'elle ne peut se constituer massivement d'esclaves et par la faible résistance des citoyens de couleur.

Armer massivement les esclaves pour étendre la liberté

À Saint-Domingue, une toute autre politique a été menée. Le 21 juin 1793, le commissaire civil Sonthonax affranchit tous les esclaves combattant pour la République. Le 29 août 1793, il proclame l'abolition de l'esclavage dans la province du Nord de Saint-Domingue. Son collègue Polverel fait de même pour les provinces de l'Ouest et du Sud, le 21 septembre 1793. Le surlendemain, Sonthonax organise l'élection des députés devant aller siéger à la Convention pour la Province du Nord. La guerre faisant rage ailleurs, il est impossible de faire la même chose. Six députés et trois suppléants sont élus, parmi eux, le noir Belley, le sang-mêlé Mills et le blanc Dufay. Ces trois hommes sont admis à siéger à la Convention le 3 février 1794. Le lendemain, l'esclavage est aboli. Le Comité de Salut public espère pouvoir conserver ses colonies et aussi détruire l'empire colonial britannique: "Qui pourra garantir les colonies anglaises de l'influence du décret de la liberté des noirs? » écrit-il le 17 février $1794^{43}$. Pour Yves Benot, l'abolition de l'esclavage n'a pas été décidée seulement pour des questions de défense nationale. L'argument militaire, selon lui, est utilisé de manière tactique et c'est une volonté idéologique du courant abolitionniste qui aboutit au texte du 16 pluvôse an II (4 février 1794) ${ }^{44}$.

Seule la situation de Saint-Domingue est évoquée lors des débats sur la liberté générale, celle de la Guadeloupe n'intervient pas. Cette île du Vent a réussi à se préserver de troubles serviles importants, par contre l'île est tombée sous la domination anglaise en avril 1794. Lors de la capitulation, les citoyens de la Guadeloupe sont sûrement informés, par des voies officieuses, du décret d'abolition. En préférant capituler sans vraiment combattre plutôt que de lutter contre un ennemi qui seul peut garantir le maintien de l'esclavage, ils révèlent leur hostilité à l'application du décret émancipateur. Dans cette circonstance, Thyrus Pautrizel, maire de Trois-Rivières avait considéré «le danger de l'ennemi comme moins fort que celui d'une insurrection d'esclaves dans l'intérieur $»^{45}$. Si la connaissance du décret d'abolition, dont les Britanniques ont certainement fait la publicité auprès des propriétaires d'esclaves de Guadeloupe, est un des éléments explicatifs de la passivité des citoyens à se défendre en avril 1794, il constitue néanmoins une arme formidable pour les commissaires civils de la Convention envoyés aux îles du Vent, Victor Hugues et Pierre Chrétien.

La reconquête de la Guadeloupe par une armée majoritairement servile

19 L'expédition part de l'île de Ré, le 23 avril 1794. La petite armée d'un millier d'hommes et les commissaires civils sont chargés d'appliquer l'abolition de l'esclavage dans les îles $\mathrm{du}$ Vent (Guadeloupe, Martinique et Sainte-Lucie). Le 3 juin 1794, la flottille française est en vue de la Guadeloupe. Le débarquement s'opère à la Pointe des Salines à Gosier 
dans la nuit du 3 au 4. Surpris par la soudaineté de l'assaut, les Britanniques abandonnent leur position en Grande-Terre ${ }^{46}$. Le 7 juin 1794, les commissaires de la Convention Pierre Chrétien et Victor Hugues proclament l'abolition de l'esclavage. Les anciens esclaves deviennent alors des citoyens français ${ }^{47}$. Le lendemain, les envoyés de la métropole invitent les "citoyens de toute couleur» de Pointe-à-Pitre et des communes adjacentes à s'enrôler en qualité de volontaires nationaux soldés pour former plusieurs bataillons ${ }^{48}$. Les anciens esclaves qui avaient auparavant la possibilité de devenir libres par leur service armé doivent désormais combattre pour conserver leur liberté.

Le 19 juin, les Britanniques contre-attaquent. Le 2 juillet, les Britanniques sont repoussés. La victoire a été assurée par 200 républicains d'Europe, une quinzaine de patriotes du pays et « environ cent affricains animés du sentiment de la liberté » selon Victor Hugues qui accable les habitants de la colonie qui l'ont majoritairement abandonné ${ }^{49}$. Du côté des républicains, les pertes sont très lourdes. Selon le capitaine d'artillerie Pélardy qui devient chef de la force armée, le 5 juillet 1794, suite aux décès de tous les autres généraux, «tous les Sans Culottes arrivés avec la Station avaient été moissonnés, ils ne nous restait pour ainsi dire que des troupes de nouvelles levées et il n'existait parmi elles aucune organisation ». Le chef de bataillon Boudet est chargé d'exercer les nouvelles recrues, essentiellement d'anciens esclaves, qui reçoivent leur instruction sous le feu de l'artillerie anglaise qui bombarde Pointe-à-Pitre ${ }^{50}$. Le décret d'abolition de l'esclavage permet pour la première fois de recruter massivement des soldats dans la masse de la population de couleur. La réaction des anciens esclaves au décret émancipateur est conforme à l'espérance de Victor Hugues.

«Beaucoup ont pris le parti des armes et ce sont montrer digne de combattre pour la liberté. Vous verrez par l'état ci joint la perte immense que nous avons fait en hommes. J'ai pris le parti de former trois bataillons dans lesquels j'ai amalgamé tous les sans culottes. Ce mélange a produit le meilleur effet possible sur l'esprit des ci devant esclave. Je leur ai accordé la même paye que les troupes de France dans les colonies. Ils s'exercent deux fois par jour et sont fort adroit au maniement des armes. Ils sont infiniment flattés d'être traités comme nos frères les sans culottes $»^{51}$.

21 Victor Hugues choisit d'amalgamer troupes blanches et noires car « les noirs seuls sans Européens ne se battroient jamais bien $»^{52}$. Les premières sont réduites à environ 200 hommes ${ }^{53}$. Elles sont renforcées par quelques patriotes réfugiés dans les îles neutres et surtout par deux à trois mille nègres et sang-mêlé. Quelques hommes du bataillon des sans culottes sont employés en qualité de sous-officiers dans les deux nouveaux bataillons pour servir d'instructeurs aux nouveaux citoyens. Le 19 juillet, Victor Hugues harangue ces nouvelles recrues.

«Vous, citoyens noirs qui, reconnaissant des bienfaits de la nation française, avez partagé nos succès en combattant pour votre liberté, imitez vos frères les Sansculottes; ils vous montreront toujours le chemin de la victoire, et consolideront avec vous votre liberté et celle de vos enfants $»^{54}$.

De juillet à septembre, la fièvre jaune s'abat sur l'armée anglaise. En effet, son quartier général est situé dans le camp de Berville, environné de marécages. La majorité des troupes du camp sont malades. Pendant la période cyclonique, la flotte britannique va s'abriter en Martinique et desserre son blocus maritime de Port de la Liberté. Les républicains en profitent pour lancer leur offensive sur la Basse-Terre. Dans la nuit du 27 au 28 septembre 1794, les républicains passent à l'offensive. Pour la première fois, les troupes noires sont confrontées directement au feu de l'ennemi. Victor Hugues 
souligne l'héroïsme des soldats noirs : «Dans cette journée mémorable, je vis pour la millième fois ce que pouvait l'amour de la liberté sur l'esprit de nos frères affricains. Je fus obligé d'aller sur le champ de bataille et leur ordonner la retraite au nom de la République; car tous voulaient mourir pour venger leurs camarades. On ne déploya jamais autant de valeur et d'héroïsme ${ }^{55}$. Le 7 octobre, les Anglais capitulent avec la possibilité de retourner dans leur pays et livrent aux républicains les royalistes qui ont combattu à leurs côtés. Le 11 décembre 1794, à l'exception des Saintes et de SaintMartin, l'ensemble de la Guadeloupe est reconquise. Avec un bataillon de sans-culottes et le décret émancipateur, le commissaire de la Convention a réussi à vaincre la coalition formée par les Anglais et les planteurs royalistes. La fièvre jaune a fortement aidé les républicains en décimant les Anglais tout en épargnant les troupes recrutées sur place déjà immunisées.

L'expansion de la liberté dans la Caraïbe

23 Le soldat de couleur, fort de ses victoires, sait qu'il peut garantir la sécurité de sa famille par le port des armes. L'idée d'Ancien Régime du service armé donnant la liberté est remplacée par la volonté de conserver et d'étendre cette même liberté par les armes. Les soldats de l'armée de Guadeloupe sont les plus visés par la propagande des agents du pouvoir. Victor Hugues leur adresse de nombreuses proclamations les incitant à étendre la liberté dans les autres colonies occupées par les Anglais. Il s'adresse aux esclaves de la Martinique en ces termes:

«Et vous, que des lois barbares ont réduits à l'esclavage, envisagez la liberté qui vous est offerte, considérez l'état actuel de ceux qui, comme vous, à la Guadeloupe et à Sainte-Lucie, gémissoient dans les mêmes liens; voyez-les devenus libres, défenseur de la patrie, et recueillant les fruits de la victoire. Empressez-vous de partager leur bonheur ; réunissez-vous sous les drapeaux de la république ; méritez les faveurs et les bienfaits qu'elle cherche à répandre sur vous $»^{56}$.

Victor Hugues mène une active propagande en direction des colonies françaises prises par les Britanniques en 1794 (Sainte-Lucie et Martinique) et même en 1763 (SaintVincent, Dominique et Grenade). Cette politique connaît un certain succès et l'expansion révolutionnaire gagne Sainte-Lucie grâce à l'homme de couleur Joseph Lambert qui s'introduit, le 15 avril 1795, à Sainte-Lucie avec le décret de la liberté générale, des armes et des munitions. Il suscite un soulèvement général des esclaves de Sainte-Lucie ${ }^{57}$. L'action de Joseph Lambert est bientôt secondée par l'arrivée du troisième bataillon et du bataillon des Antilles. Les armées révolutionnaires vont également occuper temporairement Grenade, Saint-Vincent, Saint-Martin et SainteCroix.

L'armée, fer de lance de la liberté, devient aussi le lieu de l'apprentissage de l'égalitarisme républicain. À propos des soldats noirs, Victor Hugues écrit : « Ils ont été traités comme les blancs dans le militaire, surtout, ils furent incorporés du moment de mon arrivée, il y a cinq ans; le riche propriétaire est à côté de son ci-devant esclave dans les rangs. Plus de la moitié des officiers sont noirs ou de couleur, et presque tous les fils d'habitants sont soldats. La discipline n'a jamais été mieux observée et jamais dans le militaire, il n'y a eu une seule discussion de couleur : ils sont tous citoyens, et je suis convaincu que le mot de Monsieur n'a jamais été prononcé par aucun individu de l'armée des Antilles $\aleph^{58}$. Au sein de l'armée, pour la première fois des hommes de couleur commandent à des blancs. bientôt un effet pervers. Ces dernières commencent à recruter massivement des 
troupes noires. Un émigré français déclare en 1796: "Il faut des corps noirs pour conquérir des colonies défendues par des corps noirs $»^{59}$. L'ampleur des recrutements ennemis est confirmée par les Hugues et Lebas qui affirment que les Anglais ont enrégimenté 8000 à 10000 noirs «ces êtres malheureux et abusés combattent pour river les fers sous le poids desquels ils gémissent ». L'armée ennemie compte 20000 hommes, dont 10 à 12000 à Saint-Domingue ${ }^{60}$. L'expédition de 1795-1796, commandée par le général Abercombry et l'amiral Christian est la plus grosse que l'Angleterre ait jamais envoyée outre-mer. Entre 1793 et 1801, 43750 soldats blancs anglais perdent la vie dans les Antilles (soit plus de la moitié des effectifs envoyés) et sans doute plus de 20000 marins. Beaucoup meurent des maladies tropicales ${ }^{61}$. Le recours à des «blackmen" ou troupes de couleur est rendu inéluctable pour contrer l'expansion révolutionnaire. L'abolition française de l'esclavage a des conséquences sur la société esclavagiste des colonies britanniques obligée d'émanciper les soldats esclaves enrôlés. Indirectement, la liberté générale permet un mouvement significatif d'émancipation dans les colonies ennemies.

Cette idée d'utiliser des hommes de couleur pour défendre les colonies remonte au début du conflit franco-anglais à la fin du XVII e siècle. Depuis la Guerre de Sept Ans, les besoins militaires ont crû et les affranchissements par la voie militaire aussi. Dans la conscience des esclaves, le service dans une force ou dans une cause armée quelle qu'elle soit (volontaires libres, gardes nationales, mouvement de révolte en faveur d'une faction) est fortement associé à la liberté. C'est pour cette raison qu'ils n'hésitent pas à s'enrôler dans les différentes armées qu'elles soient royalistes ou républicaines en Guadeloupe, en Martinique et à Saint-Domingue. Finalement, les esclaves après avoir analysé les forces en présence choisissent la République lorsqu'elle prend le parti d'abolir l'esclavage. Désormais tous les esclaves devenus citoyens libres se servent de leurs armes pour garantir leur liberté. Ce qui fonde la citoyenneté des anciens esclaves est à la fois le décret du 16 pluviôse an II, mais aussi leur lutte dans les rangs de la force armée recrutée dans la colonie. C'est pour cette raison que ces militaires de couleur refuseront d'être désarmés à Saint-Domingue et en Guadeloupe en 1802. Ils font de la conservation de leurs armes un préalable au maintien de leur liberté.

\section{NOTES}

1.Le terme homme de couleur englobe à la fois les libres de couleur et les esclaves.

2.C. BERNAND et A. STELLA (coord.), D'esclaves à soldats, miliciens et soldats d'origine servile XIII-XVIII ' siècles, Paris, L'Harmattan, 2006. E. LAWAETZ, Free Coloreds in St. Croix, 1744-1816, Christiansted, 1976. A. J. KUETHE, Military Reform and Sociey in New Grenada 1773-1808, Gainesville, University Press of Florida, 1978. A. J. KuETHE, Cuba 1753-1815 : Crown, Military and Society, Knoxville.University of Tennessee Press, 1986.

3.L. ABENON, La Guadeloupe de 1671 à 1759, étude politique, économique et sociale, Paris, L'Harmattan, 1987, tome II, p. 43. En 1683, il y a une seule compagnie de troupes réglées en Guadeloupe. Deux en 1694. 
4.J.-B. LABAT, Nouveau voyage aux isles de l'Amérique, Horizons Caraïbes, 1972 (rééd. de l'ouvrage de 1742), tome II, p. 36.

5.L. ABENON, op. cit., p. 165.

6.A. Perotin-Dumon, La ville aux îles, la ville dans l'île, thèse dact., Bordeaux III, p. 640.

7.CAOM (Centre des Archives d'outre-mer), Collection Moreau de Saint-Méry. F3 236, Recueil des loix particulières à la Guadeloupe et dépendance, p. 123.

8.CAOM Collection Moreau de Saint-Méry. F3 236, Recueil des loix particulières à la Guadeloupe et dépendance p. 125-133. Ordonnance du roi rétablissant les milices en Guadeloupe le 1er septembre 1768.

9.CAOM D2 C87.

10.CAOM notariat lanaspeze. Minute $n^{\circ} 5$, vente par Daillot fils de deux esclaves, le 16 janvier 1789.

11.CAOM notariat lanaspeze. Minute $n^{\circ} 86$, déclaration de Jacques, nègre libre et charpentier, en faveur du mulâtre François, engagé dans la milice depuis 1774, le 20 juillet 1790.

12.A. PEROTIN-DUMON, op. cit., p. 641-642.

13.AN D2 C41 et A. PEROTIN-Dumon, op. cit., p. 644.

14.CAOM Bibliothèque Moreau de Saint-Méry, 2e série volume 3, pièce 11 . M. B... de S., Mémoire sur la Guadeloupe, ses Isles dépendantes, son sol, ses productions \& généralement sur toutes les parties, tant militaires que d'administration, Isles du Vent, p. 16.

15.CAOM F3 73, fol. 134, Répertoire des notions coloniales, article Armes.

16.A. PEROTIN-DUMON, op. cit., p. 639.

17.J. GARRIGUS, A Struggle for Respect : The Free Coloreds of Saint-Domingue, Johns Hopkins University Press, 1988.

18.CAOM F3 20 fol. 264. Arrêté de l'assemblée coloniale de Guadeloupe du 26 janvier 1790.

19.CAOM F3 20 fol. 281-284. Règlement sur l'armement général des citoyens rendu par l'Assemblée générale coloniale le 24 février 1790.

20.CAOM F3 234. Code de la Guadeloupe. Extrait du registre des délibération de l'assemblée générale coloniale de Guadeloupe du 2 décembre 1790.

21.AD Guadeloupe Almanach de la Guadeloupe 1792, imprimé à la fin de 1791.

22.AN, DXXV 126, dossier 1000, pièce 65. Lettre des citoyens (patriotes) de Basse-Terre à l'assemblée coloniale à la fin de septembre 1791 dans laquelle ils déclarent soutenir la pétition des libres de couleur.

23.A. LACOUR, Histoire de la Guadeloupe, Basse-Terre, 1857, tome II, p. 115.

24.Musée de Chartres. Fonds Bouge. Registre des délibérations de l'assemblée coloniale. Arrêté du 30 novembre 1792.

25.AN, DXXV 125, dossier 992, pièce 9. Séance du comité de l'assemblée coloniale le 1er décembre 1792.

26.A. LACOUR, op. cit., tome II, p. 127-128.

27.G.H.V. Collot, Précis des événemens qui se sont passés à la Guadeloupe, p. 4.

28.CAOM Bibliothèque Moreau de Saint-Méry, 2ème série volume 31 , pièce $6 \mathrm{~d}$. BENOISTCAVAY et B. POHL, Rapport fait à la Commission générale extraordinaire de la Guadeloupe de leur tournée à la Grande-Terre, Basse-Terre, Bénard, 8 avril 1793, 28 p.

29.AN, DXXV 122, dossier 965, pièce 6. Force publique de la paroisse de Petit Canal le 7 mai 1793. 
30.CAOM C7A46. Fol. 62. Lettre de Collot au ministre de la Marine et des colonies du 10 avril 1793 écrite de Basse Terre.

31.AD Guadeloupe notariat dethunnes. Minute $n^{\circ} 24$, « Abandon fait à la République par le citoyen Taillandier de son mulâtre Charles », le 10 mai 1793.

32.AN, DXXV 123, dossier 973, pièce 108. Proclamation de la loi du 4 avril 1792 par Lacrosse en Guadeloupe le 29 janvier 1793 à Pointe-à-Pitre.

33. Extrait du registre des délibérations de la commission générale extraordinaire du 18 mai 1793 et arrêté concernant les hommes ayant huit années de service approuvé par Collot. CAOM C7A46. F 137. La pétition des citoyens Antoine, Sicile, Félix dit Magoti et Julien qui ont servi plus de huit ans dans la milice, puis dans la garde nationale entraîne cet arrêté.

34.Lettre écrite par le gouverneur Collot à la Commission générale et extraordinaire le 5 avril 1793 de Basse-Terre. CAOM C7A46. $\mathrm{F}^{\circ} 78$.

35.CAOM C7A46. $\mathrm{F}^{\circ}$ 103. «Instructions provisoires du citoyen gouverneur à toutes les municipalités \& commandans de la force armée, pour le moment de la guerre seulement ». Signé Collot, 1er mai 1793, Basse-Terre.

36.CAOM C7A77, Fol.49.

37.CAOM Bibliothèque Moreau de Saint-Méry, 2ème série, volume 31 , pièce $6 t$. Extrait des registres des délibérations de l'Assemblée organisatrice provisoirement administrative du 20 ventôse an II (10 mars 1794).

38.G.H.V. Collot, Précis des événemens qui se sont passés à la Guadeloupe, p. 23.

39.AN, AD VII $21 C, n^{\circ} 45$. Extraits des registres de la Société des Amis de la République française du 17 pluviôse an II (5 février 1794).

40.CAOM C7A47. Fol. 78.

41.Joseph Leborgne, second de Rochambeau en Martinique, est ensuite renvoyé en France à la fin de 1793.

42.Y. BENOT, La Révolution française et la fin des colonies, 1989, p. 175-176.

43. CAOM C7 A 47, fol. 126-127. Extrait des registres du Comité de Salut Public du 25 pluviôse an II (17 février 1794), supplément aux instructions données par le Comité de Salut Public aux commissaires nationaux envoyés dans les Isles du Vent.

44.Y. BENOT, « Comment la convention a-t-elle voté l'abolition de l'esclavage ? », dans Révolutions aux colonies, Société des Études Robespierristes, 1993, p. 25.

45.AN C7A47. Fol. 66. Délibération des corps constitués, 29 germinal an II (18 avril 1794).

46.CAOM C7 A 47, fol. 106. Lettre de Leissègues commandant de la station des Iles du Vent du 28 prairial an II (16 juin 1794).

47.CAOM C7 A 47, fol. 8. Proclamation des commissaires de la Convention Pierre Chrétien et Victor Hugues du 19 prairial an II (7 juin 1794).

48.CAOM C7 A 47, fol. 9. Proclamation des commissaires Pierre Chrétien et Victor Hugues, le20 prairial an II (8 juin 1794).

49.CAOM F3 237 Événements historiques Guadeloupe. Lettre de Victor Hugues au président de la Convention nationale du 26 frimaire an III (16 décembre 1794).

50.CAOM F3 237 Événements historiques Guadeloupe. Précis des événements qui se sont passés à la Guadeloupe, le 2 floréal an III (21 avril 1795).

51. CAOM C7 A 47, fol. 20-25. Lettre de Victor Hugues du 4 thermidor an II (22 juillet 1794) au Comité de Salut Public.

52.Ibidem. 
53.SHD Armée de terre B9-2. Lettre de Victor Hugues du 4 thermidor an II (22 juillet 1794) au président de la Convention nationale. « Nous sommes réduits au quart par les morts et les blessés ». Le bataillon des sans-culottes compte 800 hommes à son débarquement en Guadeloupe.

54.SHD Armée de terre B9-2. Adresse de Victor Hugues aux Républicains des armées de terre et de mer de la république, actuellement à la Guadeloupe du 1er thermidor an 2 (19 juillet 1794).

55.CAOM F3 237 Événements historiques Guadeloupe. Lettre de Victor Hugues au président de la convention nationale du 26 frimaire an III (16 décembre 1794).

56.Centre des archives diplomatiques de Nantes, Guadeloupe Correspondance volume 2. Proclamation des commissaires délégués de la Convention aux habitants de la Martinique, le 21 frimaire an IV (12 décembre 1795).

57.CAOM B.M.S. 1ère série volume 173. Courrier de la France et des colonies $n^{\circ} 25,12$ novembre 1795. Précis de la prise de Sainte-Lucie.

58.AN, C7A 50, fol. 16-19. Lettre de Victor Hugues au ministre du 23 prairial an VI (11 juin 1798).

59. Collection Marcel Chatillon. Lettre de Faudoas à de Curt expédiée de Saint-Pierre, le 6 août 1796.

60.AN, C7A 49, fol. 12-13. Lettre des agents au ministre de la Marine du 24 floréal an IV(1er mai 1796).

61.M. DuFFY, Soldier, Sugar and Seapower : the British Expeditions to the West Indies and the War Against Revolutionary France, Clarendon Press, 1987, 420 p.

\section{RÉSUMÉS}

L'idée de donner des armes aux esclaves n'est pas née des conflits politiques révolutionnaires. Des compagnies de couleur ont été formées dans toute l'Amérique coloniale au XVIII ${ }^{\mathrm{e}}$ siècle ${ }^{2}$. Dans l'affrontement séculaire entre la France et le Royaume-Uni commencé en 1666, les colonies deviennent un enjeu de plus en plus important. En 1666, le gouverneur de la Martinique arme un certain nombre d'esclaves pour défendre la colonie. Très vite, l'idée de recourir à des esclaves pour assurer la défense des territoires coloniaux s'impose. Mais si l'armement des esclaves donne des bras supplémentaires pour la protection des possessions outre-mer, dans le même temps, il enlève des bras à la culture. Le service armé entraîne à plus ou moins long terme, comme dans la Rome antique, l'affranchissement. Quels effets l'armement d'esclaves a-t-il sur le développement de la liberté et les mentalités serviles?

The Arming of Men of Color and Liberty in the French West Indies: the case of Guadeloupe during the old Regime and the Revolution. The idea of arming slaves was not born of the political conflicts of the French Revolution. Companies of men of color had been formed throughout colonial America in the eighteenth century. During the spectacular confrontation between France and Britain that began in 1666, the colonies became an increasingly important issue. Rapidly, the idea of resorting to the use of slaves to ensure the defence of colonial territories suggested itself. But if the arming of slaves provided additional men for the protection of overseas possessions, at the same time it deprived farming of necessary manpower. This 
armed service was more or less long-term, and as in Ancient Rome, it resulted in freedom. What effects did the arming of slaves have on the development of liberty and on slave mentalities?

INDEX

Mots-clés : esclavage, soldats, affranchissement, libres de couleur, milice

\section{AUTEUR \\ FRÉDÉRIC RÉGENT}

Université des Antilles et de la Guyane EA 929, Campus du Camp Jacob, Rue des officiers, 97120 Saint-Claude, Guadeloupe, regentfrederic@wanadoo.fr 